\title{
Supraphos: A Supramolecular Strategy to Prepare Bidentate Ligands.
}

Vincent F. Slagt, Marc Röder, Paul C. J. Kamer, Piet W. N. M. van Leeuwen, Joost N. H. Reek.* Van 't Hoff Institute for Molecular Sciences, University of Amsterdam, Nieuwe Achtergracht 166, 1018 WV Amsterdam (The Netherlands), Fax: (+31)20-525-6422

General Procedures. Unless stated otherwise, reactions were carried out under an atmosphere of argon using standard Schlenk techniques. THF, hexane and diethyl ether were distilled from sodium benzophenone ketyl, $\mathrm{CH}_{2} \mathrm{Cl}_{2}$, isopropanol and methanol were distilled from $\mathrm{CaH}_{2}$ and toluene was distilled from sodium under nitrogen. NMR spectra $\left({ }^{1} \mathrm{H},{ }^{31} \mathrm{P}\right.$ and $\left.{ }^{13} \mathrm{C}\right)$ were measured on a Bruker DRX $300 \mathrm{MHz}$ and Varian Mercury 300 $\mathrm{MHz} ; \mathrm{CDCl}_{3}$ was used as a solvent, if not further specified. Mass spectra were recorded on a JEOL JMS SX/SX102A four sector mass spectrometer; for FAB-MS 3-nitrobenzyl alcohol was used as matrix. UV-vis spectroscopy experiments were performed on a HP 8453 UV/Visible System. Elemental analyses were obtained on an Elementar Vario EL apparatus. Gas chromatographic analyses were run on an Interscience HR GC Mega 2 apparatus (split/splitless injector, J\&W Scientific, DB-1 J\&W 30m column, film thickness $3.0 \mu \mathrm{m}$, carrier gas 70kPa He, FID Detector) equipped with a Hewlett_packard Data system (Chrom-Card). Chiral HPLC analyses were carried out using a Daicel Chiralcel-OD column $(0.46$ x $25 \mathrm{~cm})$.

Materials. With exception of the compounds given below, all reagents were purchased from commercial suppliers and used without further purification. Diisopropylethylamine and triethylamine were distilled from $\mathrm{CaH}_{2}$ under argon. The following compound were synthesized according to published procedures: phosphorochloridites, ${ }^{1}$ pyridylphosphines $\mathbf{b}$ and $\mathbf{c},{ }^{2}$ phosphines $\mathbf{d - f},{ }^{3}$ hydroxyl porphyrins ${ }^{4}$ and zinc(II) porphyrins were prepared according to the method of Adler. ${ }^{5}$

Synthesis of 5-(2-hydroxyphenyl)-10,15,20-tris(phenyl) porphyrin

Salicylaldehyde $(200 \mathrm{mmol})$ and benzaldehyde $(200 \mathrm{mmol})$ were dissolved in $1.0 \mathrm{l}$ of propionic acid and heated till reflux. Under an air flow and vigorous stirring pyrrole $(400 \mathrm{mmol})$ was added and the solution was refluxed for 1 hour. The reaction mixture was cooled to $60{ }^{\circ} \mathrm{C}$ and $200 \mathrm{ml}$ of methanol was added. The reaction was stored overnight at $4{ }^{\circ} \mathrm{C}$, allowing the porphyrin to precipitate. The reaction mixture was filtered and washed several times with methanol until the filtrate was colorless. The porphyrin was purified using column chromatography (basic alumina, $\mathrm{CH}_{2} \mathrm{Cl}_{2}$, upgrade $1 \% \mathrm{MeOH}$ in $\mathrm{CH}_{2} \mathrm{Cl}_{2}$ ), giving 5-(2-hydroxyphenyl)-10,15,20-tris(phenyl) porphyrin (1.05 g, $1.66 \mathrm{mmol}, 1.7 \%)$.

Synthesis of 5-(3-hydroxyphenyl)-10,15,20-tris(phenyl) porphyrin

3-hydroxybenzaldehyde $(6.35 \mathrm{~g}, 52.0 \mathrm{mmol})$ and benzaldehyde $(15.8 \mathrm{ml}, 156 \mathrm{mmol})$ were dissolved in $750 \mathrm{ml}$ of propionic acid and heated till reflux. Under an air flow and vigorous stirring pyrrole $(14.4 \mathrm{ml}, 208 \mathrm{mmol})$ was added and the solution was refluxed for 1 hour. The reaction mixture was cooled to $60{ }^{\circ} \mathrm{C}$ and $100 \mathrm{ml}$ of methanol was added. The reaction was stored overnight at $4{ }^{\circ} \mathrm{C}$, allowing the porphyrin to precipitate. The reaction mixture was filtered and washed several times with methanol until the filtrate was colorless. The porphyrin was purified using column chromatography (basic alumina, $\mathrm{CH}_{2} \mathrm{Cl}_{2}$, upgrade $2 \% \mathrm{MeOH}$ in $\mathrm{CH}_{2} \mathrm{Cl}_{2}$ ), giving 5-(3hydroxyphenyl)-10,15,20-tris(phenyl) porphyrin (1.09 g, $1.73 \mathrm{mmol}, 3.3 \%)$.

Synthesis of (3,3'-5,5'-tetra-tert-butyl-1,1'-biphenyl-2,2'-diyl)-(5-(phenyl-2-yl)-10,15,20-tris(phenyl)-zinc(II) porphyrin) phosphite 1

5-(2-hydroxyphenyl)-10,15,20-tris(phenyl)-zinc(II) porphyrin (1.59 g, $2.30 \mathrm{mmol}$ ), azeotropically dried with toluene $(3 \times 5 \mathrm{ml})$, and diisopropylethylamine $(4.0 \mathrm{ml}, 23.0 \mathrm{mmol})$ were dissolved in THF $(80 \mathrm{ml})$ and the solution was cooled to $0{ }^{\circ} \mathrm{C}$. Freshly prepared 3,3'-5,5'-tetra-tert-butyl-1,1'-biphenyl-2,2'-diyl phosphorochloridite $(0.73 \mathrm{~g}$, $2.09 \mathrm{mmol})$ was dissolved in THF $(20 \mathrm{ml})$ and added dropwise, stirring was continued for 15 minutes. The 
cooling bath was removed and the solution was allowed to warm to room temperature, stirring was continued for 30 minutes. The reaction mixture was filtered and the solvent evaporated. The crude product was purified by flash column chromatography under Argon (basic alumina; $\mathrm{CH}_{2} \mathrm{Cl}_{2}$ ) to remove the excess of hydroxyl-porphyrin, giving $1(0.887 \mathrm{~g}, 0.88 \mathrm{mmol}, 42 \%)$ as a purple-red solid: ${ }^{1} \mathrm{H}$ NMR $(300 \mathrm{MHz}): \delta 8.90(\mathrm{~m}, 8 \mathrm{H}), 8.22(\mathrm{~m}, 6 \mathrm{H}), 8.09$ $(\mathrm{m}, 1 \mathrm{H}), 7.76(\mathrm{~m}, 9 \mathrm{H}), 7.64(\mathrm{~m}, 1 \mathrm{H}) 7.59(\mathrm{~m}, 1 \mathrm{H}), 7.47((\mathrm{~m}, 1 \mathrm{H}), 7.19(\mathrm{~d}, 2 \mathrm{H}, \mathrm{J}=4.2 \mathrm{~Hz}), 6.93(\mathrm{~d}, 2 \mathrm{H}, \mathrm{J}=4.2 \mathrm{~Hz})$, 1.21 (s, 18H), 0.93 (s, 18H); ${ }^{31} \mathrm{P}$ NMR (121.5 MHz): $\delta 133.4 ;{ }^{13} \mathrm{C}-\mathrm{ATP}(75.465 \mathrm{MHz}): \delta 150.67,150.41,150.32$, 146.63, 145.53, 143.35, 135.89, 134.71, 132.46, 132.46, 132.05, 129.27, 127.61, 126.96, 126.72, 126.68, 122.56, 121.85, 121.07, 35.24, 31.62, 30.95; HRMS (FAB+): m/z calcd. for $\mathrm{C}_{72} \mathrm{H}_{68} \mathrm{~N}_{4} \mathrm{O}_{3} \mathrm{PZn}\left(\left[\mathrm{MH}^{+}\right]\right): 1131.4320$; obsd.: 1131.4314; anal. calcd. for $\mathrm{C}_{72} \mathrm{H}_{67} \mathrm{~N}_{4} \mathrm{O}_{3} \mathrm{PZn}$ : C, 76.35; H, 5.96; N, 4.95. Found: C, 76.26; H, 6.15; N, 5.06.

Synthesis of (3,3'-5,5'-tetra-tert-butyl-1,1'-biphenyl-2,2'-diyl)-(5-(phenyl-3-yl)-10,15,20-tris(phenyl)-zinc(II) porphyrin) phosphite 2

This compound was prepared as described for 1, using 5-(3-hydroxyphenyl)-10,15,20-tris(phenyl)-zinc(II) porphyrin. Yield $(47 \%)$ as a purple-red solid: ${ }^{1} \mathrm{H}$ NMR $(300 \mathrm{MHz}): \delta 8.96-8.94(\mathrm{~m}, 8 \mathrm{H}), 8.25-8.20(\mathrm{~m}, 6 \mathrm{H}), 8.01$ $(\mathrm{s}, 1 \mathrm{H}), 7.95(\mathrm{~d}, 1 \mathrm{H}, \mathrm{J}=8.7 \mathrm{~Hz}), 7.82-7.77(\mathrm{~m}, 9 \mathrm{H}), 7.67-7.62(\mathrm{~m}, 1 \mathrm{H}), 7.49-7.45(\mathrm{~m}, 1 \mathrm{H}), 7.42(\mathrm{~s}, 2 \mathrm{H}), 7.23(\mathrm{~s}$, 2H), $1.50-1.47(\mathrm{~m}, 18 \mathrm{H}), 1.36-1.32(\mathrm{~s}, 18 \mathrm{H}) ;{ }^{31} \mathrm{P}$ NMR $(121.5 \mathrm{MHz}): \delta 139.02 ;{ }^{13} \mathrm{C}-\mathrm{ATP}(75.465 \mathrm{MHz}): 150.77$ (C), 150.48 (C), 150.22 (C), 146.98 (C), 144.66 (C), 143.04 (C), 140.42 (C), 137.02_(C), 136.09_(C), 134.67 $(\mathrm{CH}), 133.07(\mathrm{C}), 132.23(\mathrm{CH}), 130.68(\mathrm{CH}), 129.12(\mathrm{CH}), 128.45(\mathrm{CH}), 127.75(\mathrm{CH}), 126.80(\mathrm{CH}), 154.54$ $(\mathrm{CH}), 125.07(\mathrm{CH}), 124.60(\mathrm{CH}), 122.56(\mathrm{C}), 122.39(\mathrm{C}), 122.13(\mathrm{C}), 121.41(\mathrm{C}), 119.73(\mathrm{CH}), 35.68(\mathrm{C}), 34.85$ (C), $31.70(\mathrm{CH}), 31.47(\mathrm{CH})$; HRMS $(\mathrm{FAB}+): \mathrm{m} / \mathrm{z}$ calcd. for $\mathrm{C}_{72} \mathrm{H}_{68} \mathrm{~N}_{4} \mathrm{O}_{3} \mathrm{PZn}\left(\left[\mathrm{MH}^{+}\right]\right)$: 1131.4321 ; obsd.: 1131.4329; anal. calcd. for $\mathrm{C}_{72} \mathrm{H}_{67} \mathrm{~N}_{4} \mathrm{O}_{3} \mathrm{PZn}$ : C, 76.35; H, 5.96; N, 4.95. Found: C, 76.83; H, 6.35; N, 4.68.

Synthesis of (S)-(1,1'-binaphthyl-2,2'-diyl)-(5-(phenyl-2-yl)-10,15,20-tris(phenyl)-zinc(II) porphyrin) phosphite 3

5-(2-hydroxyphenyl)-10,15,20-tris(phenyl)-zinc(II) porphyrin $(1.59 \mathrm{~g}, 2.30 \mathrm{mmol})$, azeotropically dried with toluene $(3 \times 5 \mathrm{ml})$, and diisopropylethylamine $(4.0 \mathrm{ml}, 23.0 \mathrm{mmol})$ were dissolved in THF $(80 \mathrm{ml})$ and the solution was cooled to $-40^{\circ} \mathrm{C}$. Freshly prepared (S)-1,1'-binaphthyl-2,2'-diyl phosphorochloridite $(0.73 \mathrm{~g}, 2.09 \mathrm{mmol})$ was dissolved in THF $(20 \mathrm{ml})$ and added dropwise, stirring was continued for 15 minutes. The cooling bath was removed and the solution was allowed to warm to room temperature, stirring was continued for 30 minutes. The reaction mixture was filtered and the solvent evaporated. The crude product was purified by flash column chromatography under Argon (basic alumina; $\mathrm{CH}_{2} \mathrm{Cl}_{2}$ ) to remove the excess of hydroxyl-porphyrin, giving 3 $(0.887 \mathrm{~g}, 0.88 \mathrm{mmol}, 42 \%)$ as a purple-red solid: ${ }^{1} \mathrm{H}$ NMR $(300 \mathrm{MHz}): \delta 8.94(\mathrm{~d}, 6 \mathrm{H}, \mathrm{J}=4.5 \mathrm{~Hz}), 8.89(\mathrm{~d}, 1 \mathrm{H}$, $\mathrm{J}=4.5 \mathrm{~Hz}), 8.84(\mathrm{~d}, 1 \mathrm{H}, \mathrm{J}=4.5 \mathrm{~Hz}), 8.23(\mathrm{~m}, 6 \mathrm{H}), 8.13(\mathrm{~m}, 2 \mathrm{H}), 7.77(\mathrm{~m}, 11 \mathrm{H}), 7.66(\mathrm{~m}, 2 \mathrm{H}), 7.55(\mathrm{~m}, 1 \mathrm{H}), 7.16(\mathrm{~m}$, $1 \mathrm{H}), 6.91(\mathrm{~d}, 1 \mathrm{H}, \mathrm{J}=8.7 \mathrm{~Hz}), 6.84(\mathrm{~m}, 1 \mathrm{H}), 6.60(\mathrm{~d}, 1 \mathrm{H}, \mathrm{J}=8.1 \mathrm{~Hz}), 6.45(\mathrm{~m}, 2 \mathrm{H}), 6.38(\mathrm{~m}, 1 \mathrm{H}), 5.72(\mathrm{~d}, 1 \mathrm{H}, \mathrm{J}=$ 8.7Hz), $5.60(\mathrm{~d}, 1 \mathrm{H}, \mathrm{J}=8.4 \mathrm{~Hz}) ;{ }^{31} \mathrm{P}$ NMR $(121.5 \mathrm{MHz}): \delta 145.15 ;{ }^{13} \mathrm{C}-\mathrm{ATP}(75.465 \mathrm{MHz}): \delta 150.62,150.50$, $150.22,146.43,145.32,143.25,135.79,134.71,132.42,132.12,131.50-130.22,128.61,127.53-126.63,125.50$, 125.21, 122.56, 121.85, 121.07; HRMS (FAB+): m/z calcd. for $\mathrm{C}_{64} \mathrm{H}_{40} \mathrm{~N}_{4} \mathrm{O}_{3} \mathrm{PZn}\left(\left[\mathrm{MH}^{+}\right]\right): 1007.2130$; obsd.: 1007.2144; anal. calcd. for $\mathrm{C}_{64} \mathrm{H}_{39} \mathrm{~N}_{4} \mathrm{O}_{3} \mathrm{PZn}$ : C, 76.23; H, 3.90; N, 5.56. Found: C, 76.08; H, 4.16; N, 5.42.

Synthesis of (R)-(1,1'-binaphthyl-2,2'-diyl)-(5-(phenyl-2-yl)-10,15,20-tris(phenyl)-zinc(II) porphyrin) phosphite 4

This compound was prepared as described for 3, using freshly prepared (R)-1,1'-binaphthyl-2,2'-diyl phosphorochloridite. Yield $(46 \%)$ as a purple-red solid: ${ }^{1} \mathrm{H}$ NMR $(300 \mathrm{MHz}): \delta 8.94(\mathrm{~d}, 6 \mathrm{H}, \mathrm{J}=4.5 \mathrm{~Hz}), 8.89(\mathrm{~d}$, $1 \mathrm{H}, \mathrm{J}=4.5 \mathrm{~Hz}), 8.84(\mathrm{~d}, 1 \mathrm{H}, \mathrm{J}=4.5 \mathrm{~Hz}), 8.22(\mathrm{~m}, 6 \mathrm{H}), 8.14(\mathrm{~m}, 2 \mathrm{H}), 7.78(\mathrm{~m}, 11 \mathrm{H}), 7.64(\mathrm{~m}, 2 \mathrm{H}), 7.55(\mathrm{~m}, 1 \mathrm{H}), 7.16$ $(\mathrm{m}, 1 \mathrm{H}), 6.91(\mathrm{~d}, 1 \mathrm{H}, \mathrm{J}=8.4 \mathrm{~Hz}), 6.84(\mathrm{~m}, 1 \mathrm{H}), 6.60(\mathrm{~d}, 1 \mathrm{H}, \mathrm{J}=8.1 \mathrm{~Hz}), 6.45(\mathrm{~m}, 2 \mathrm{H}), 6.38(\mathrm{~m}, 1 \mathrm{H}), 5.71(\mathrm{~d}, 1 \mathrm{H}, \mathrm{J}=$ $8.7 \mathrm{~Hz}), 5.59(\mathrm{~d}, 1 \mathrm{H}, \mathrm{J}=8.4 \mathrm{~Hz}) ;{ }^{31} \mathrm{P}$ NMR $(121.5 \mathrm{MHz}): \delta 145.13 ;{ }^{13} \mathrm{C}-\mathrm{ATP}(75.465 \mathrm{MHz}): \delta 150.62,150.50$, $150.22,146.43,145.32,143.25,135.79,134.71,132.42,132.12,131.50-130.22,128.61,127.53-126.63,125.50$, 125.21, 122.56, 121.85, 121.07; HRMS (FAB+): m/z calcd. for $\mathrm{C}_{64} \mathrm{H}_{40} \mathrm{~N}_{4} \mathrm{O}_{3} \mathrm{PZn}\left(\left[\mathrm{MH}^{+}\right]\right)$: 1007.2130 ; obsd.: 1007.2136; anal. calcd. for $\mathrm{C}_{64} \mathrm{H}_{39} \mathrm{~N}_{4} \mathrm{O}_{3} \mathrm{PZn}$ : C, 76.23; H, 3.90; N, 5.56. Found: C, 76.29; H, 4.14; N, 5.22.

Synthesis of (S)-(1,1'-binaphthyl-2,2'-diyl)-(5-(phenyl-3-yl)-10,15,20-tris(phenyl)-zinc(II) porphyrin) phosphite 5 
This compound was prepared as described for 3, using 5-(3-hydroxyphenyl)-10,15,20-tris(phenyl)-zinc(II) porphyrin and freshly prepared (S)-1,1'-binaphthyl-2,2'-diyl phosphorochloridite. Yield (39\%) as a purple-red

\section{S3}

solid: ${ }^{1} \mathrm{H}$ NMR (300 MHz): $\delta$ 8.97-8.94 (m, 6H), 8.83-8.78 (m, 2H), 8.24-8.21 (m, 6H), 8.04-7.79 (m, 6H), 7.77 (s, 9H) 7.70-7.15 (m, 10H); ${ }^{31} \mathrm{P}$ NMR (121.5 MHz): $\delta 142.49 ;{ }^{13} \mathrm{C}-\mathrm{ATP}(75.465 \mathrm{MHz}): \delta 150.53(\mathrm{C}), 150.28(\mathrm{C})$, $144.83(\mathrm{C}), 143.01(\mathrm{C}), 134.67(\mathrm{CH}), 132.39(\mathrm{CH}), 132.02(\mathrm{CH}), 131.49(\mathrm{C}), 131.05(\mathrm{CH}), 130.76(\mathrm{CH}), 130.23$ $(\mathrm{CH}), 128.61(\mathrm{CH}), 127.83(\mathrm{CH}), 127.31(\mathrm{CH}), 127.26(\mathrm{CH}), 126.83(\mathrm{CH}), 126.71(\mathrm{CH}), 126.63(\mathrm{CH}), 125.50$ $(\mathrm{CH}), 125.23(\mathrm{CH}), 121.99(\mathrm{CH}), 121.49(\mathrm{C}), 120.19(\mathrm{CH})$; HRMS (FAB+): m/z calcd. for $\mathrm{C}_{64} \mathrm{H}_{40} \mathrm{~N}_{4} \mathrm{O}_{3} \mathrm{PZn}$ $\left(\left[\mathrm{MH}^{+}\right]\right):$1007.2129; obsd.: 1007.2109; anal. calcd. for $\mathrm{C}_{64} \mathrm{H}_{39} \mathrm{~N}_{4} \mathrm{O}_{3} \mathrm{PZn}: \mathrm{C}, 76.23 ; \mathrm{H}, 3.90 ; \mathrm{N}$, 5.56. Found: C, 76.36; H, 4.08; N, 5.42 .

Synthesis of (S)-(3,3'-bis(trimethylsilyl)-1,1'-binaphthyl-2,2'-diyl)-(5-(phenyl-2-yl)-10,15,20-tris(phenyl)-zinc(II) porphyrin) phosphite 6

This compound was prepared as described for 3, using freshly prepared (S)-3,3'-bis(trimethylsilyl)-1,1'binaphthyl-2,2'-diyl phosphorochloridite. Yield (51\%) as a purple-red solid: ${ }^{1} \mathrm{H}$ NMR $(300 \mathrm{MHz}): \delta 9.06(\mathrm{~d}, 2 \mathrm{H})$, $8.96(\mathrm{~d}, 2 \mathrm{H}, \mathrm{J}=5.4 \mathrm{~Hz})), 8.85(\mathrm{~d}, 2 \mathrm{H}, \mathrm{J}=5.1 \mathrm{~Hz}), 8.83(\mathrm{~m}, 2 \mathrm{H}), 8.66(\mathrm{~m}, 1 \mathrm{H}), 8.49(\mathrm{~m}, 3 \mathrm{H}), 8.23(\mathrm{~m}, 2 \mathrm{H}), 8.12(\mathrm{~m}$, $2 \mathrm{H}), 8.02(\mathrm{~m}, 1 \mathrm{H}), 7.94(\mathrm{~d}, 2 \mathrm{H}, \mathrm{J}=5.4 \mathrm{~Hz}), 7.84-7.55(\mathrm{~m}, 9 \mathrm{H}), 7.45(\mathrm{~m}, 1 \mathrm{H}), 7.37(\mathrm{~m}, 2 \mathrm{H}), 7.22-7.11(\mathrm{~m}, 4 \mathrm{H})$, $6.63(\mathrm{~m}, 1 \mathrm{H}), 8.94(\mathrm{~m}, 1 \mathrm{H}), 5.06(\mathrm{~m}, 1 \mathrm{H}), 4.50(\mathrm{~m}, 1 \mathrm{H}), 0.51(\mathrm{~s}, 9 \mathrm{H}), 0.03(\mathrm{~s}, 9 \mathrm{H}) ;{ }^{31} \mathrm{P}$ NMR $(121.5 \mathrm{MHz}): \delta$ $150.03 ;{ }^{13} \mathrm{C}$-ATP $(75.465 \mathrm{MHz}): \delta 150.7,150.5,150.4,145.4,135.7,134.7,132.4,132.1,131.5-130.2,128.7$, 127.6-126.5, 125.4, 125.1, 122.5, 121.9, 121.1, 0.35, 0.20; HRMS (FAB+): m/z calcd. for $\mathrm{C}_{70} \mathrm{H}_{56} \mathrm{~N}_{4} \mathrm{O}_{3} \mathrm{PSi}_{2} \mathrm{Zn}$ ([MH $\left.{ }^{+}\right]$: 1151.2930; obsd.: 1151.2920; anal. calcd. for $\mathrm{C}_{70} \mathrm{H}_{55} \mathrm{~N}_{4} \mathrm{O}_{3} \mathrm{PSi}_{2} \mathrm{Zn}: \mathrm{C}, 72.93 ; \mathrm{H}, 4.81 ; \mathrm{N}, 4.86$. Found: $\mathrm{C}$, 72.96; H, 4.83; N, 4.71 .

Synthesis of (S)-(1,1'-binaphthyl-2,2'-diyl)-(3-pyridyl) phosphite $\mathbf{g}$

3-Hydroxypyridine $(1.44 \mathrm{~g}, 15.1 \mathrm{mmol})$, azeotropically dried with toluene $(3 \times 5 \mathrm{ml})$, and triethylamine $(2.3 \mathrm{ml}$, $16.6 \mathrm{mmol})$ were dissolved in THF $(40 \mathrm{ml})$ and the solution was cooled to $-40{ }^{\circ} \mathrm{C}$. Freshly prepared (S)-1,1'binaphthyl-2,2'-diyl phosphorochloridite $(5.3 \mathrm{~g}, 15.1 \mathrm{mmol})$ was dissolved in THF $(20 \mathrm{ml})$ and added dropwise. The cooling bath was removed and the solution was allowed to warm to room temperature, stirring was continued for 1 hour. The reaction mixture was filtered and the solvent evaporated. A mixture of toluene/hexane 1/3 (40 ml)

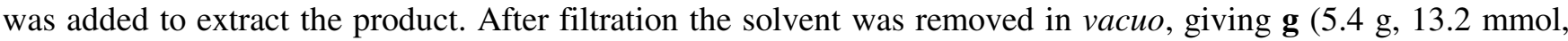
$87 \%$ ) as a white solid: ${ }^{1} \mathrm{H}$ NMR $(300 \mathrm{MHz}): \delta 8.54(\mathrm{~d}, 1 \mathrm{H}, \mathrm{J}=2.4 \mathrm{~Hz}), 8.40(\mathrm{~d}, 1 \mathrm{H}, \mathrm{J}=4.2 \mathrm{~Hz}), 8.02(\mathrm{~d}, 1 \mathrm{H}, \mathrm{J}=$ $8.7 \mathrm{~Hz}), 7.95(\mathrm{~d}, 1 \mathrm{H}, \mathrm{J}=8.7 \mathrm{~Hz}), 7.92(\mathrm{~d}, 2 \mathrm{H}, \mathrm{J}=8.7 \mathrm{~Hz}), 7.56-7.16(\mathrm{~m}, 10 \mathrm{H}) ;{ }^{31} \mathrm{P}$ NMR $(121.5 \mathrm{MHz}): \delta 143.05$; ${ }^{13} \mathrm{C}(75.465 \mathrm{MHz}): 148.88\left(\mathrm{~d}, \mathrm{~J}_{\mathrm{cp}}=6.1 \mathrm{~Hz}\right), 147.52\left(\mathrm{~d}, \mathrm{~J}_{\mathrm{cp}}=4.8 \mathrm{~Hz}\right), 146.84\left(\mathrm{~d}, \mathrm{~J}_{\mathrm{cp}}=1,4 \mathrm{~Hz}\right), 145.80(\mathrm{~s}), 142.71(\mathrm{~d}$, $\left.\mathrm{J}_{\mathrm{cp}}=7.3 \mathrm{~Hz}\right), 133.02(\mathrm{~s}), 132.75(\mathrm{~s}), 132.01(\mathrm{~s}), 131.55(\mathrm{~s}), 130.97(\mathrm{~s}), 130.36(\mathrm{~s}), 129.29(\mathrm{~s}), 128.67\left(\mathrm{~d}, \mathrm{~J}_{\mathrm{cp}}=\right.$ 4.8Hz), 128.48 (s), 127.945 (s), 127.83 (s), 127.28 (s), 127.19 (s), 126.80 (s), 126.67 (s), 125.68 (s), 125.49 (s), 124.49 (s) 121.77 (s), 121.59 (s). HRMS (FAB+): m/z calcd. for $\mathrm{C}_{25} \mathrm{H}_{17} \mathrm{NO}_{3} \mathrm{P}\left(\left[\mathrm{MH}^{+}\right]\right): 410.0946$; obsd.: 410.0952; anal. calcd. for $\mathrm{C}_{25} \mathrm{H}_{16} \mathrm{NO}_{3} \mathrm{P}: \mathrm{C}, 73.35 ; \mathrm{H}, 3.94$; N, 3.42. Found: $\mathrm{C}, 73.20 ; \mathrm{H}, 4.16$; N, 3.25 .

\section{Synthesis of (R)-(1,1'-binaphthyl-2,2'-diyl)-(3-pyridyl)phosphite $\mathbf{h}$}

This compound was prepared as described for $\mathbf{g}$, using 5-(3-hydroxyphenyl)-10,15,20-tris(phenyl)-zinc(II) porphyrin and freshly prepared (R)-1,1'-binaphthyl-2,2'-diyl phosphorochloridite. Yield (78\%) as a purple-red solid: ${ }^{1} \mathrm{H}$ NMR $(300 \mathrm{MHz}): \delta 8.54(\mathrm{~d}, 1 \mathrm{H}, \mathrm{J}=2.4 \mathrm{~Hz}), 8.40(\mathrm{~d}, 1 \mathrm{H}, \mathrm{J}=4.2 \mathrm{~Hz}), 8.02(\mathrm{~d}, 1 \mathrm{H}, \mathrm{J}=8.7 \mathrm{~Hz}), 7.95(\mathrm{~d}$, $1 \mathrm{H}, \mathrm{J}=8.7 \mathrm{~Hz}), 7.92(\mathrm{~d}, 2 \mathrm{H}, \mathrm{J}=8.7 \mathrm{~Hz}), 7.56-7.16(\mathrm{~m}, 10 \mathrm{H}) ;{ }^{31} \mathrm{P}$ NMR $(121.5 \mathrm{MHz}): \delta 143.05 ;{ }^{13} \mathrm{C}-\mathrm{ATP}(75.465$ $\mathrm{MHz}): 148.88\left(\mathrm{~d}, \mathrm{~J}_{\mathrm{cp}}=6.1 \mathrm{~Hz}\right), 147.52\left(\mathrm{~d}, \mathrm{~J}_{\mathrm{cp}}=4.8 \mathrm{~Hz}\right), 146.84\left(\mathrm{~d}, \mathrm{~J}_{\mathrm{cp}}=1,4 \mathrm{~Hz}\right), 145.80(\mathrm{~s}), 142.71\left(\mathrm{~d}, \mathrm{~J}_{\mathrm{cp}}=7.3 \mathrm{~Hz}\right)$, $133.02(\mathrm{~s}), 132.75(\mathrm{~s}), 132.01(\mathrm{~s}), 131.55(\mathrm{~s}), 130.97(\mathrm{~s}), 130.36(\mathrm{~s}), 129.29(\mathrm{~s}), 128.67$ (d, J $\left.\mathrm{J}_{\mathrm{cp}}=4.8 \mathrm{~Hz}\right), 128.48(\mathrm{~s})$, 127.945 (s), 127.83 (s), 127.28 (s), 127.19 (s), 126.80 (s), 126.67 (s), 125.68 (s), 125.49 (s), 124.49 (s) 121.77 (s), 121.59 (s). HRMS (FAB+): m/z calcd. for $\mathrm{C}_{25} \mathrm{H}_{17} \mathrm{NO}_{3} \mathrm{P}\left(\left[\mathrm{MH}^{+}\right]\right): 410.0946$; obsd.: 410.0938; anal. calcd. for $\mathrm{C}_{25} \mathrm{H}_{16} \mathrm{NO}_{3} \mathrm{P}: \mathrm{C}, 73.35 ; \mathrm{H}, 3.94 ; \mathrm{N}, 3.42$. Found: $\mathrm{C}, 73.64 ; \mathrm{H}, 4.38 ; \mathrm{N}, 3.05$.

Synthesis of (S)-(3,3'-bis(trimethylsilyl)-1,1'-binaphthyl-2,2'-diyl)-(3-pyridyl) phosphite $\mathbf{i}$

This compound was prepared as described for $\mathbf{g}$, using freshly prepared (S)-3,3'-bis(trimethylsilyl)-1,1'binaphthyl-2,2'-diyl phosphorochloridite. Yield (66\%) as a white solid: ${ }^{1} \mathrm{H}$ NMR $(300 \mathrm{MHz}): \delta 8.30(\mathrm{~d}, 1 \mathrm{H}, \mathrm{J}=$ 
$2.7 \mathrm{~Hz}), 8.27(\mathrm{~d}, 1 \mathrm{H}, \mathrm{J}=5.1 \mathrm{~Hz}), 8.08(\mathrm{~d}, 2 \mathrm{H}, \mathrm{J}=2.7 \mathrm{~Hz}), 7.92(\mathrm{dd}, 2 \mathrm{H}, \mathrm{J}=2.7,8.1 \mathrm{~Hz}), 7.45-7.40(\mathrm{~m}, 2 \mathrm{H}), 7.39-$ $7.31(\mathrm{~m}, 1 \mathrm{H}), 7.26-7.09(\mathrm{~m}, 5 \mathrm{H}), 0.43(\mathrm{~s}, 9 \mathrm{H}), 0.38(\mathrm{~s}, 9 \mathrm{H}) ;{ }^{31} \mathrm{P}$ NMR $(121.5 \mathrm{MHz}): \delta$ 138.99; HRMS (FAB+):

\section{S4}

m/z calcd. for $\mathrm{C}_{31} \mathrm{H}_{33} \mathrm{NO}_{3} \mathrm{PSi}_{2}\left(\left[\mathrm{MH}^{+}\right]\right)$: 554.1737; obsd.: 554.1725; anal. calcd. for $\mathrm{C}_{31} \mathrm{H}_{32} \mathrm{NO}_{3} \mathrm{PSi}_{2}: \mathrm{C}, 67.24 ; \mathrm{H}$, 5.82; N, 2.53. Found: C, 67.18; H, 5.89; N, 2.42.

\section{UV-vis spectroscopy titration experiments}

In a typical experiment the UV-vis cuvette was filled with $2.0 \mathrm{ml}$ of $7.5 \times 10^{-5} \mathrm{M}$ phosphite zinc(II) porphyrin in freshly distilled toluene. Subsequently small aliquots of a toluene solution containing $1.5 \times 10^{-3} \mathrm{M}$ pyridylphosphine (in the presence or absence of $\mathrm{HRh}\left(\mathrm{PPh}_{3}\right)_{3} \mathrm{CO}$ ) and $7.5 \times 10^{-5} \mathrm{M}$ phosphite zinc(II) porphyrin was added stepwise. Measurements were performed at room temperature. In the UV-vis spectrum the typical decrease of the Q-bands of the porphyrin at $551 \mathrm{~nm}$ and $591 \mathrm{~nm}$ were monitored. We have analyzed the titration curves with a fitting program developed by Hunter et al.: Bisson, A. P.; Hunter, C. A.; Carlos, J.; Young, K. Chem. Eur. J. 1998, 4, 845 .

The binding study of in the presence of $\mathrm{HRh}\left(\mathrm{PPh}_{3}\right)_{3} \mathrm{CO}$ comprises a competition experiment: the first triphenylphosphine will exchange with the phosphite because of the stronger coordination properties. The coordination of ligand $\mathbf{b}$ has to compete with triphenylphosphine, and the chelate effect will promote ligand $\mathbf{b}$ compared to triphenylphosphine. The chelate energy is therefore expressed in an increase in binding energy, which is found to correspond to $7 \mathrm{~kJ} / \mathrm{mol}$ (binding energies were $20.5 \mathrm{~kJ} / \mathrm{mol}$ and $27.5 \mathrm{~kJ} / \mathrm{mol}$ respectively).

\section{High-pressure NMR-experiments}

In a typical experiment the high pressure NMR tube was filled with $(30 \mu \mathrm{mol})$ of $\left[\mathrm{Rh}(\mathrm{acac})(\mathrm{CO})_{2}\right],(75 \mu \mathrm{mol})$ of phosphite zinc(II) porphyrin, $(75 \mu \mathrm{mol})$ phosphine and $1.5 \mathrm{ml}$ of toluene- $\mathrm{d}_{8}$. The tube was purged three times with 15 bar of $\mathrm{CO} / \mathrm{H}_{2}(1: 1)$, pressurized to approximately 20 bar, heated to $80^{\circ} \mathrm{C}$ and incubated for 1 hour. Measurements were performed at $25^{\circ} \mathrm{C}$.

Table 1. Selected ${ }^{1} \mathrm{H}$ - and ${ }^{31} \mathrm{P}-\mathrm{NMR}$ data of high-pressure NMR-spectroscopy of various metal complexes at $\mathrm{T}=$

\begin{tabular}{ccccccc}
\hline Complex & $\begin{array}{c}\delta\left({ }^{31} \mathrm{P}\right) \text {-phosphine } \\
(\mathrm{ppm})\end{array}$ & $\begin{array}{c}\delta\left({ }^{31} \mathrm{P}\right) \text {-phosphite } \\
(\mathrm{ppm})\end{array}$ & $\begin{array}{c}\mathrm{J}_{\mathrm{Rh}-\mathrm{P}} \\
(\mathrm{Hz})\end{array}$ & $\begin{array}{c}\mathrm{J}_{\mathrm{Rh}-\mathrm{PO} 3} \\
(\mathrm{~Hz})\end{array}$ & $\begin{array}{c}\mathrm{J}_{\mathrm{P}-\mathrm{PO} 3} \\
(\mathrm{~Hz})\end{array}$ & $\begin{array}{c}\delta\left({ }^{1} \mathrm{H}\right)-\mathrm{Rh}-\mathrm{H} \\
(\mathrm{ppm})\end{array}$ \\
\hline$[\mathrm{Rh}(\mathrm{acac})(\mathrm{CO})(\mathbf{1})]^{\mathrm{a}}$ & $\mathrm{c}$ & 127.8 & $\mathrm{c}$ & 300 & $\mathrm{c}$ & $\mathrm{c}$ \\
{$\left[\mathrm{HRh}(\mathrm{CO})_{2}(\mathbf{1} \bullet \mathbf{b})\right]$} & 29.4 & 144.3 & 143 & 265 & 153 & -10.6 \\
{$\left[\mathrm{HRh}(\mathrm{CO})_{2}(\mathbf{1})(\mathbf{a})\right]$} & 30.1 & 179.8 & $\mathrm{~b}$ & $\mathrm{~b}$ & $\mathrm{~b}$ & -9.8 \\
{$\left[\mathrm{HRh}(\mathrm{CO})_{2}(\mathbf{a})_{2}\right]$} & 37.9 & $\mathrm{c}$ & 140 & $\mathrm{c}$ & $\mathrm{c}$ & -9.1 \\
{$\left[\mathrm{HRh}(\mathrm{CO})_{2}(\mathbf{2})_{2}\right]$} & $\mathrm{c}$ & 150.4 & $\mathrm{c}$ & 243 & $\mathrm{c}$ & -9.3 \\
{$\left[\mathrm{HRh}(\mathrm{CO})_{2}(\mathbf{2} \cdot \mathbf{b})\right]$} & 31.4 & 148.6 & $\mathrm{~b}$ & $\mathrm{~b}$ & $\mathrm{~b}$ & -10.8 \\
$\mathrm{PdMeCl}(\mathbf{3 \bullet b})^{\mathrm{d}}$ & 24.3 & 135.3 & - & - & 620 & - \\
\hline
\end{tabular}

(a) the $[\mathrm{Rh}(\mathrm{acac})(\mathrm{CO})(\mathbf{1})]$ was used cause, no satisfactory NMR-data were observed for the $\mathrm{HRh}(\mathrm{CO})_{3}(\mathbf{1})$, due to solubility problems, (b) broad signals were observed, (c) not present. (d) under 1 bar of nitrogen atmosphere. 
S5

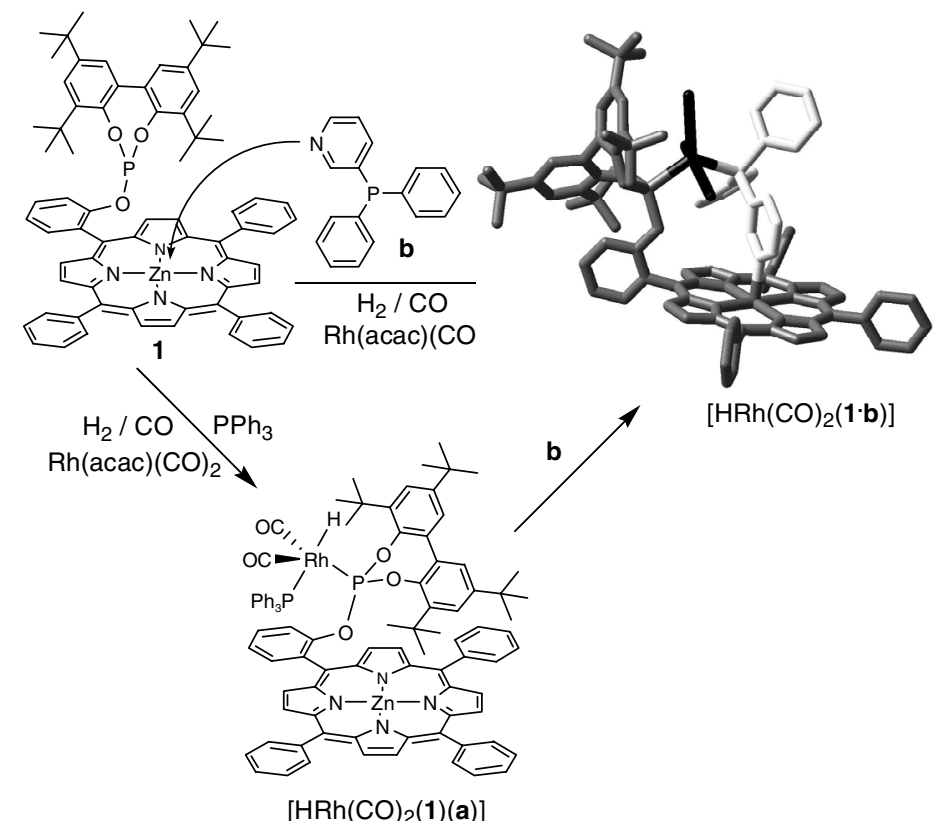

Figure 1: Schematic presentation of the possible routes to obtain the $\left[\mathrm{HRh}(\mathrm{CO})_{2}(1-b)\right]$ complex

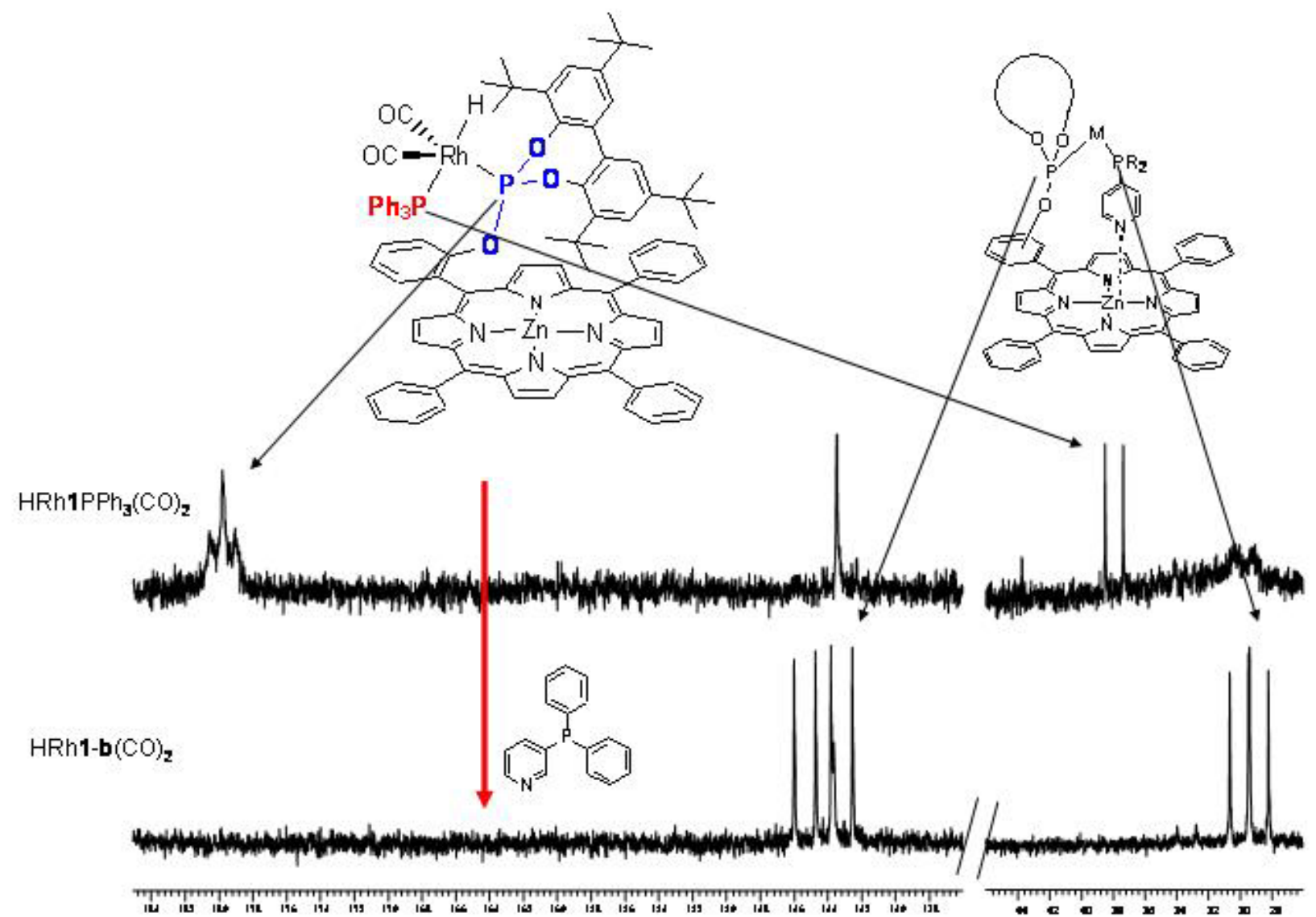


Figure 2: The ${ }^{31} \mathrm{P}-\mathrm{NMR}$ spectra of the experiment that shows the chelate effect of $\left[\mathrm{HRh}(\mathrm{CO})_{2}(\mathbf{1}-\mathbf{b})\right]$ : Starting from the triphenylphosphine complex $\left[\mathrm{HRh}(\mathrm{CO})_{2}(\mathbf{1}) \mathrm{PPh}_{3}\right]$ the $\left[\mathrm{HRh}(\mathrm{CO})_{2}(\mathbf{1 - b})\right]$ complex is quantitatively formed upon addition of $\mathbf{b}$.

\section{S6}

\section{Catalysis.}

The hydroformylation experiments were performed as follows. A stainless steel $25 \mathrm{ml}$ autoclave, equipped with a teflon stirring bar, was charged with $0.42 \mu \mathrm{mol}$ of $\left[\mathrm{Rh}(\mathrm{acac})(\mathrm{CO})_{2}\right], 10.4 \mu \mathrm{mol}$ of phosphine and $0.017 \mathrm{ml}$ of dipea in $4.0 \mathrm{ml}$ of toluene. The solution was incubated for 1 under $20 \mathrm{bar} \mathrm{CO} / \mathrm{H}_{2}(1: 1)$. The pressure was reduced to $1 \mathrm{bar}$ and a mixture of $0.34 \mathrm{ml}$ styrene and $0.17 \mathrm{ml}$ of decane in $0.67 \mathrm{ml}$ of toluene was added. Subsequently the $\mathrm{CO} / \mathrm{H}_{2}$ pressure was pressurized to 20 bar. The mixture was stirred, depending on the temperature, for 1 hour $\left(80^{\circ} \mathrm{C}\right)$ or 18 hours $\left(40^{\circ} \mathrm{C}\right)$. The autoclave was cooled down to $0^{\circ} \mathrm{C}$ in ice and the pressure was reduced to 1.0 bar. A sample was taken and the conversion was checked by GC analysis of the crude product after filtration over a plug silica to remove the catalyst.

The allylic alkylation experiments were performed as follows. Under Schlenk conditions $0.50 \mu \mathrm{mol}$ of $[\mathrm{Pd}(\text { allyl }) \mathrm{Cl}]_{2}, 3.0 \mu \mathrm{mol}$ phosphite and $3.0 \mu \mathrm{mol}$ phosphine were dissolved in $5.0 \mathrm{ml}$ of $\mathrm{CH}_{2} \mathrm{Cl}_{2}$ and stirred for 30 minutes. Respectively, $50 \mu \mathrm{mol}$ 1,3-diphenyl-allylacetate, $150 \mu \mathrm{mol}$ dimethylmalonate, $150 \mu \mathrm{mol}$ BSA and 50 $\mu \mathrm{mol}$ decane and a pinch of KOAc were added. The mixture was stirred at $25^{\circ} \mathrm{C}\left(-20^{\circ} \mathrm{C}\right)$ and after 24 hours the reaction was stopped by adding a saturated ammoniumchloride solution of water. Subsequently, $5.0 \mathrm{ml}$ of petroleum ether was added and the solution was washed once more with a saturated $\mathrm{NH}_{4} \mathrm{Cl}$ solution. The organic phase was dried over $\mathrm{Na}_{2} \mathrm{SO}_{4}$, filtered and the conversion was checked by $\mathrm{GC}$ analysis. The solution was chromatographed $\left(\mathrm{SiO}_{2}\right.$; petroleum ether/ $\left.\mathrm{CH}_{2} \mathrm{Cl}_{2}=1 / 1\right)$ to give analytically pure products. ${ }^{6}$ Enantiomeric purities were determened by chiral HPLC (OD column, eluens $0.5 \%$ iso-propanol in hexane $t_{R}(R)=33.2$ min. and $t_{S}(S)=$ 34.9 min.).

Buisman, G. J. H.; van der Veen, L. A.; Klootwijk, A.; de Lange, W. G. J.; Kamer, P. C. J.; van Leeuwen, P. W. N. M.; Vogt, D. Organometallics 1997, 16, 2929.

2 (a) Bowen, R. J.; Garner, A. C.; Berners-Price, S. J.; Jenkins, I. D.; Sue, R. E. J. Organomet. Chem. 1998, 554, 181. (b) Buhling, A.; Kamer, P. C. J.; van Leeuwen, P. W. N. M.; J. Mol. Catal. A 1995, 98, 69.

3 (a) Kellner, K.; Hanke, W. J. Organomet. Chem. 1987, 326, C9-C12. (b) Kellner, K.; Hanke, W.; Tzschach, A. Zeitschrift fuer Chem. 1984, 24, 193. (c) Maier, L. Helv. Chim. Acta 1965, 48, 1034. (d) Bissky, G.; Roschenthaler, G.-V.; Lork, E.; Barten, J.; Medebielle, M.; Staninets, V.; Kolomeitsev, A. A. J. Fluorine Chem. 2001, 109, 173. (e) 3-pyridylmethyldiphenylphosphine and imidazol-1-yl-mehtyldiphenylphosphine were kindly donated by Shell Research and Technology center, Amsterdam, The Netherlands.

(a) Cammidge, A. N.; Lifsey, K. M. Tetrahedron Letters 2000, 41, 6655. (b) Fujii, H.; Yoshimura, T.; Kamada, H. Chem. Lett. 1996, 8, 581.

$5 \quad$ Adler, A. D.; Longo, F. R.; Kampas, F.; Kim, J. J. Inorg. Nucl. Chem. 1970, 32, 2443.

$6 \quad$ Product characterization data were found in agreement with the reported literature, see for example: (a) von Matt, P.; Loiseleur, O.; Koch, G.; Pfaltz, A.; Lefeber, C.; Feucht, T.; Helmchen, G. Tetrahedron Asymmytry 1994, 5, 573. (b) Bower, J. F.; Jumnah, R.; Williams, A. C.; Williams, J. M. J. J. Chem. Soc., Perkin Trans 1 1997, 1411. 